\title{
Macular vasculature, visual acuity, and irreversibly sickled cells in homozygous sickle cell disease
}

\author{
R. J. MARSH, S. M. FORD, M. F. RABB,${ }^{1}$ R. J. HAYES, ${ }^{2}$ AND G. R. SERJEANT \\ From the Medical Research Council Laboratories (Jamaica), University of the West Indies, Kingston, Jamaica; \\ the 'Sickle Cell Eye Unit of the University of Illinois, Eye and Ear Infirmary, Chicago, Illinois; and the \\ ${ }^{2}$ Tropical Epidemiology Unit, London School of Hygiene and Tropical Medicine
}

SUMMARY Observations of visual acuity and the conjunctival, macular, and perimacular vascularity have been assessed in patients with homozygous sickle cell (SS) disease. There were 17 matched pairs, each consisting of one patient with a high count $(>15 \%)$ and one with a low count $(<5 \%)$ of irreversibly sickled cells (ISCs). The macular vascular bed was assessed by measurements of the foveal avascular zone (FAZ), perimacular avascular zones, and counts of perimacular vascular abnormalities (perimacular counts). Small foveal avascular zones and high perimacular counts were commoner in younger than older patients and there was a significant inverse correlation between size of the FAZ and the perimacular count. These observations were compatible with the hypothesis that perimacular vessel anomalies represent the early vaso-occlusive phase which progresses to ischaemia and the formation and enlargement of avascular areas. Visual acuity was assessed by Snellen's test type and by measuring contrast sensitivity. There was no obvious relationship between acuity measured by the 2 methods and no relationship between acuity and observations of macular vascularity. High ISC counts were significantly related to abnormalities of the conjunctival vasculature, but no relationship was noted with abnormalities of the macular vasculature or with visual acuity.

The small-vessel obstruction characteristic of sickle cell disease may affect the retinal vasculature. Although peripheral retinal vessel involvement is most widely documented, capillary drop-out and vascular remodelling at the posterior pole has also been reported ${ }^{12}$ and may be associated with a reduction of visual acuity. Such macular capillary occlusion is assumed to be secondary to intravascular sickling and thus might be related to the irreversibly sickled cell (ISC) count, as in the relationship previously shown with the degree of vessel obstruction in the conjunctival vasculature. ${ }^{3}$

This hypothesis has been tested in the present study by examining the visual acuity and macular capillary vasculature in matched groups of patients with homozygous sickle cell (SS) disease with high $(\geqslant 15 \%)$ and with low $(\leqslant 5 \%)$ counts of irreversibly sickled cells.

Correspondence to Dr Graham R. Serjeant. MRC Laboratories, University of the West Indies, Kingston 7, Jamaica.

\section{Materials and methods}

Patients were selected from among adults attending the sickle cell clinic of the University Hospital of the West Indies. Twenty patients (10 male, 10 female) with SS disease and with steady-state ISC counts of $15 \%$ or above were selected at random from clinic files and matched by age and sex with 20 patients in whom ISC counts were $5 \%$ or below. The ISC count is thought to reflect the degree of intravascular sickling, which may vary markedly between different patients with SS disease. Measured by a differential red cell count, ISC counts may vary from $0-40 \%$ in different patients, but within individual patients the counts oscillate within a relatively narrow range characteristic of the individual patient.

All patients were given appointments for ophthalmological examination during the 3-week period of the study, but of these only 8 pairs of male patients and 9 pairs of female patients actually attended. Ages ranged from 20 to 49 years with a mean age of 30 
Fig. 1 Fluorescein angiogram of perimacular region in a 23-year-old female patient demonstrating enlarged FAZ, microaneurysm (a). perimacular avascular zone (b), and hairpin loop (c).

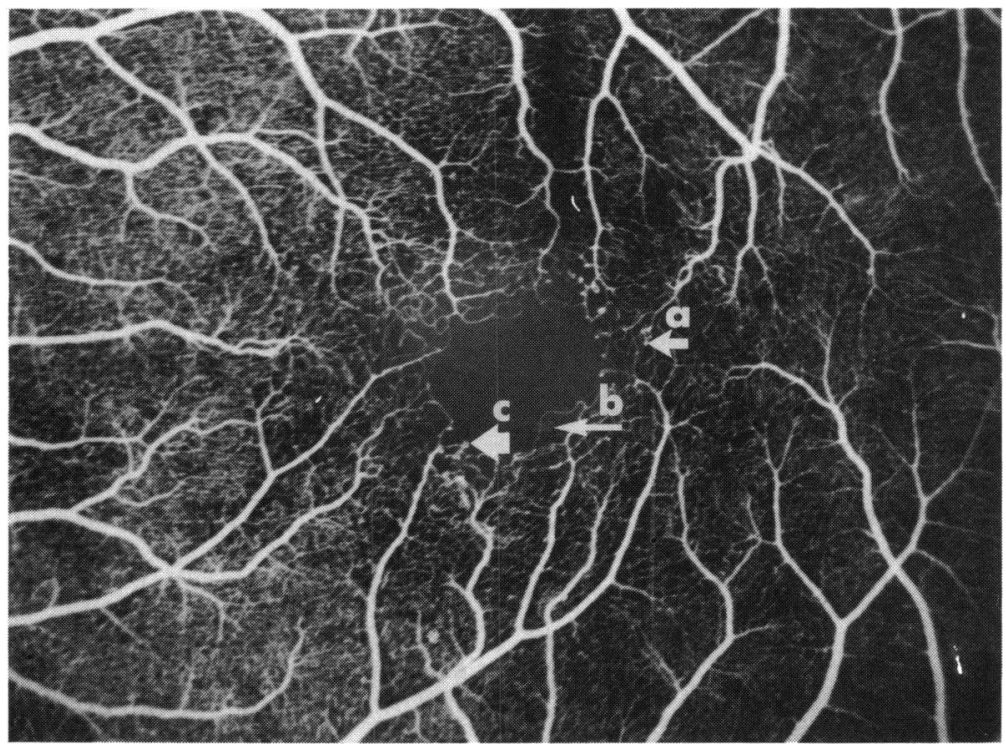

years. The diagnosis of SS disease was based on the finding of a single major haemoglobin band in the position of $\mathrm{HbS}$ on haemoglobin electrophoresis on cellulose acetate and agar gel, compatible $\mathrm{HbA}_{2}$ levels, and family study where possible.

Visual acuity was assessed as best corrected vision with Snellen's test type. Contrast sensitivity was assessed with plates 2-7 of the Arden gratings, ${ }^{4}$ greater contrast being gradually exposed successively until the grating was detected by the patient. The reading was scored at this point and the scores of all 6 plates added to give a total score for each eye. The conjunctival vasculature was assessed in all quadrants of each eye with the use of a photoslit lamp, and the vessel anomalies were graded according to the classification of Serjeant $e t$ al. $^{3}$ The optic discs and maculae of both eyes were photographed in colour with a Zeiss retinal camera and $\times 2$ lens, and the films were examined for abnormal vessels. Angiography of the macular areas of both eyes was performed and the angiograms examined on a Zeiss Dokumator with a $\times 17.5$ lens to determine the foveal and perimacular avascular zones and the number of perimacular capillary abnormalities. The foveal avascular zone was measured as the mean of vertical and horizontal diameters and expressed as an average for both eyes. Perimacular avascular zones (Fig. 1) were assessed in an arbitrary circle of $2.4 \mathrm{~mm}$ diameter centred on the macula. They were arbitrarily graded into scores $0-3$ varying from no detectable capillary drop-out to moderate capillary drop-out, and the scores for each pair of eyes were added to give a total score for each patient. Perimacular capillary abnormalities (peri- macular counts) were assessed within the same $2.4 \mathrm{~mm}$ circle by counting the number of microaneurysms, dilated capillaries, dark and enlarged arteriolar segments, and hairpin-shaped vascular loops (Fig. 1,2). The total count of abnormalities in each pair of eyes was then calculated.

All assessments were performed by one ophthalmologist (R.J.M.) without knowledge of the ISC count.

\section{Results}

VISUAL ACUITY

Visual acuity measured by Snellen's test type was $6 / 6$ in $50 / 68(74 \%)$ eyes and $6 / 12$ or better in $65 / 68(96 \%)$ eyes. Acuity was $6 / 24$ in one eye affected by vitreous haemorrhage and 6/36 in 2 eyes with long-standing aphakia. There was a tendency for reduced acuity to be more common in older than younger patients, acuity of less than $6 / 6$ occurring in $4 / 20$ patients under 30 years compared with $8 / 14$ patients over this age. This difference just failed to reach statistical significance $(p=0 \cdot 06)$.

The Arden grating scores showed considerable variation, though there was generally good agreement between results in both eyes of the same patient. Scores varied between 57 and 114 in individual eyes (mean score 85), and pathological scores as defined by Arden and Jacobson ${ }^{5}$ in British populations ( $\left.>82\right)$ occurred in 37/67 (55\%) of eyes in this patient group. There was a tendency for scores to be higher in female patients (mean $\pm S D ; 89 \cdot 4 \pm 12 \cdot 4)$ than male 


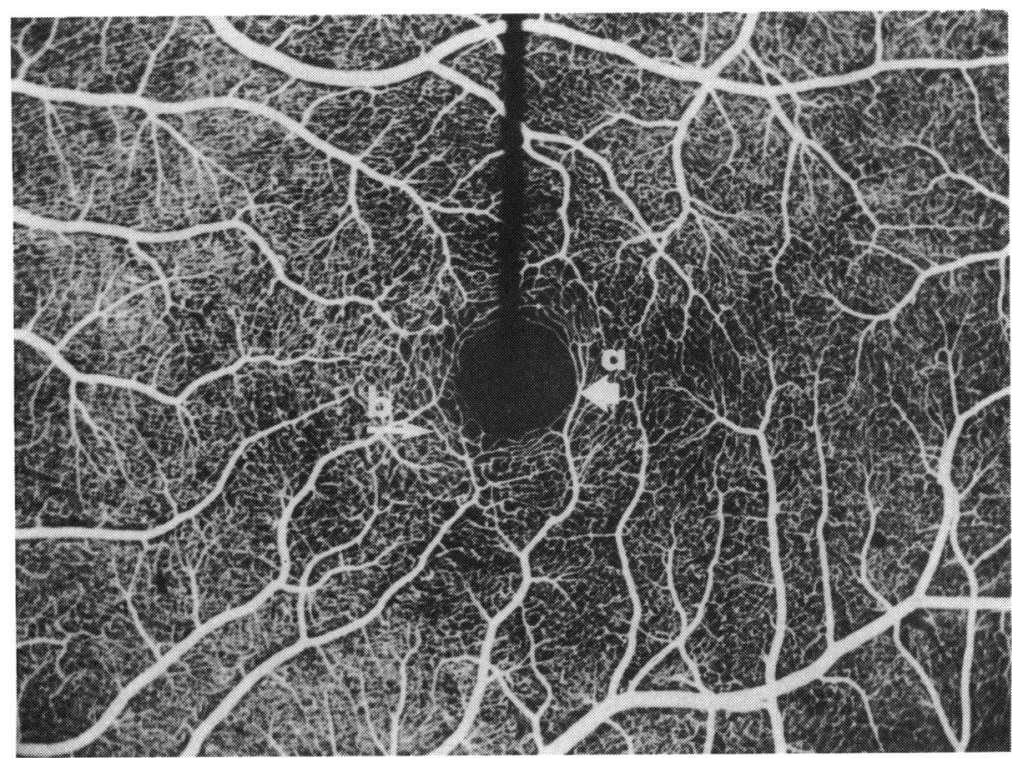

Fig. 2 Fluorescein angiogram of perimacular region in a 20 -year-old female patient demonstrating a normal FAZ but with an enlarged arteriole forming part of the perifoveolar arcade (a), and a dilated arteriolar segment (b).

$(79 \cdot 0 \pm 13 \cdot 9)$, the difference being statistically significant $(p<0 \cdot 05)$, but there was no significant relationship with age.

There was no obvious relationship between scores with the Arden gratings and with Snellen's test type (Fig. 3) and no relationship between acuity measured by either method and the ISC count.

CONJUNCTIVAL VESSEL ABNORMALITIES

There was good agreement between the scores in the quadrants of both eyes, and a total score (obtained by adding scores in all 8 quadrants) was therefore used in the statistical analysis. The previously reported positive relationship between ISC count and conjunctival vessel abnormalities was confirmed (Table 1), and an effect of age was also noted. In younger patients, who had a wide spread of scores, higher scores were associated with high ISC counts, whereas most of the older patients had high conjunctival scores regardless of their ISC count (Fig. 4). How-



Fig. 3 Comparison of visual acuity measured by Snellen's test tvpe with contrast sensitivity assessed by Arden's gratings. Each point represents one eye $(n=67)$; one eye with visual acuity of 6/24 was not assessed by Arden's gratings. 
Table 1 Comparison of contrast sensitivity, conjunctival score, and macular abnormalities in patients with high and low ISC counts

\begin{tabular}{|c|c|c|c|c|}
\hline . & $\begin{array}{l}\text { Mean of high } \\
\text { ISC group }\end{array}$ & $\begin{array}{l}\text { Mean of low } \\
\text { ISC group }\end{array}$ & $\begin{array}{l}\text { Paired } t \\
\text { statistic }\end{array}$ & Significance \\
\hline Arden gratings & $83 \cdot 4$ & $86 \cdot 0$ & -0.91 & NS \\
\hline Conjunctival score & $18 \cdot 9$ & $12 \cdot 9$ & $2 \cdot 56$ & $p<0 \cdot 05$ \\
\hline Foveal avascular zone $(\mathrm{mm})$ & 0.66 & $0 \cdot 71$ & $-1 \cdot 23$ & NS \\
\hline Perimacular avascular zones & $1 \cdot 1$ & 1.9 & $-1 \cdot 89$ & NS \\
\hline Perimacular count of abnormalities & 212 & 173 & $1 \cdot 31$ & NS \\
\hline
\end{tabular}

See text for explanation of variables. NS=not significant.

ever, sample sizes within age groups were too small for the latter observation to be statistically significant.

\section{MACULAR FINDINGS}

Accurate observations on the macular vasculature were not possible in 10 eyes because of medial opacities in 4 and poor angiographic quality in 6 . In cases where both maculae were visible there was generally good agreement between the eyes in respect of the foveal avascular zone and perimacular avascular zones, and though there was considerable variation in perimacular counts between the 2 eyes this variation was considerably less than the variation between patients. In cases where macular observations were unavailable in one eye observations on the other eye were used as best estimates of the missing observations.

The diameter of the foveal avascular zone varied from 0.46 to $1.03 \mathrm{~mm}$ and was positively correlated with the grading of perimacular avascular zones $(r=0.50 ; p<0.01)$. Perimacular counts varied widely (total counts varied from 58 to 398 ) and showed a significant negative relationship with the foveal avascular zone ( $r=-0 \cdot 46 ; p<0 \cdot 01)$ (Fig. 5).

Foveal and perimacular avascular zones tended to be larger in the low ISC group and perimacular counts to be higher in the high ISC group, but none of these relationships were statistically significant (Table 1). High perimacular counts were confined to voung patients, counts in excess of 225 occurring in 10/21 patients $\leqslant 30$ years compared with $0 / 12$ patients over this age $(p<0 \cdot 01$; Fisher's exact test $)$. Small foveal avascular zones were also more common in voung patients, a mean diameter of $<0.65 \mathrm{~mm}$ occurring in $9 / 21$ patients $\leqslant 30$ years compared with $1 / 12$ patients over this age, but the difference was not significant.

None of the above macular findings showed significant relationships with conjunctival vessel abnormalities or with visual acuity assessed by either Snellen's test type or the Arden gratings.

OPTIC DISC

Saccular dilatations of small vessels on the optic disc occurred in $11 / 17(65 \%)$ patients in the high ISC group compared with $7 / 17(41 \%)$ patients in the low ISC group $\left(\chi^{2}=1 \cdot 06 ;\right.$ not significant $)$.
Fig. 4 The relationship of total conjunctival score and age in patients with high (๑) and low (O) ISC counts.

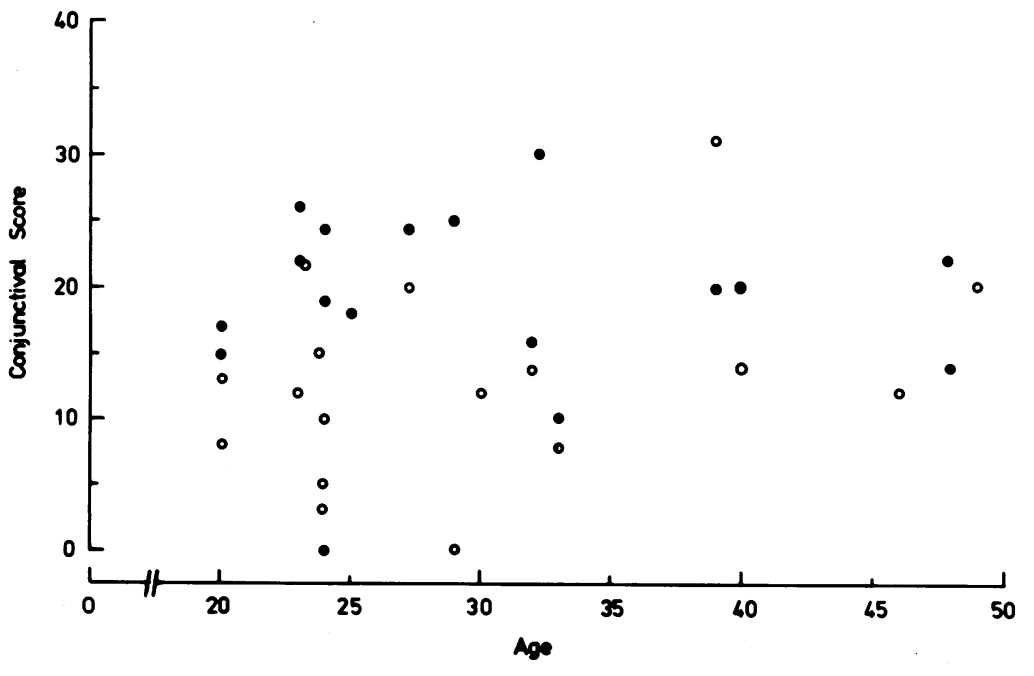




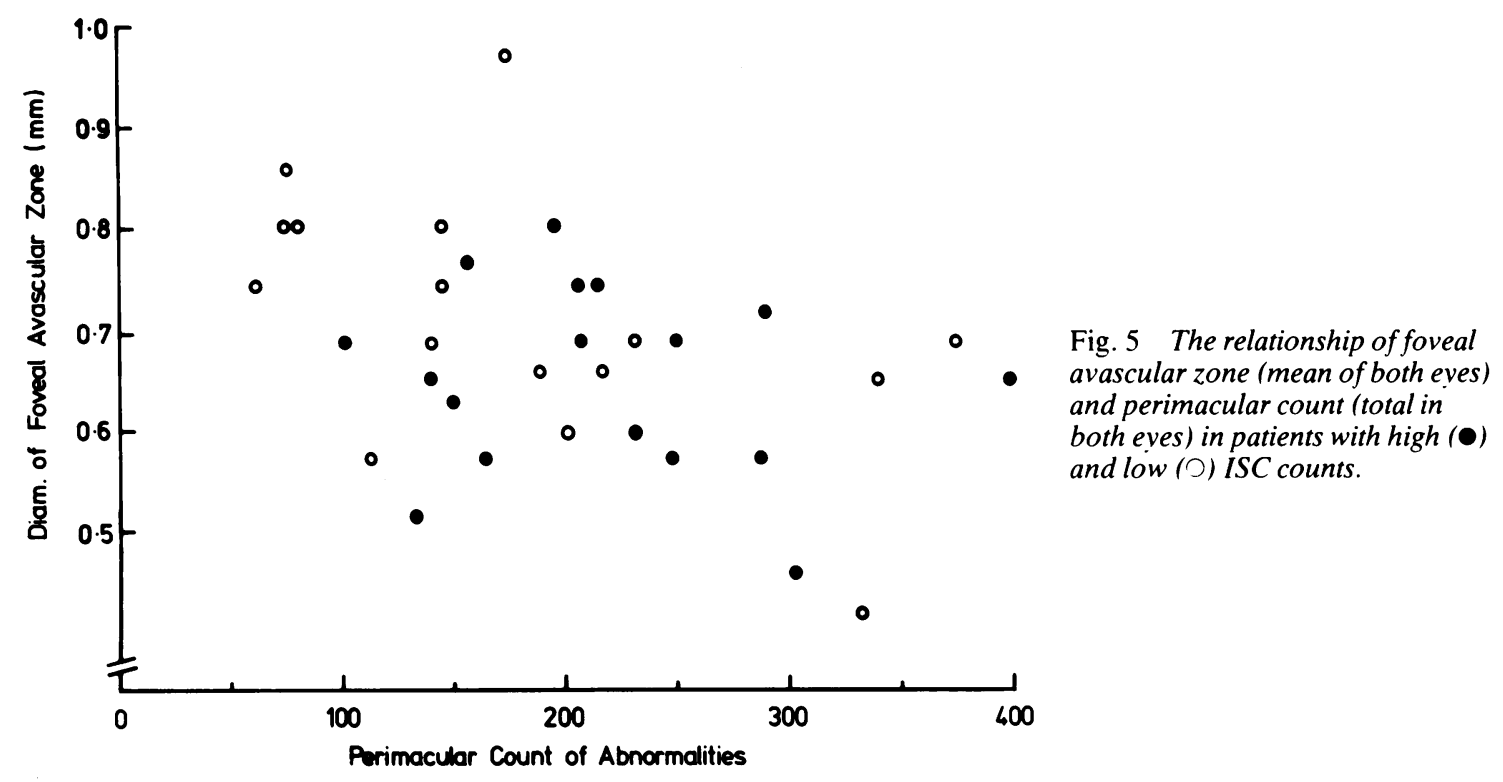

\section{Discussion}

The previously reported association between the number of irreversibly sickled cells (ISC count) and the density of abnormalities in the conjunctival vasculature has been confirmed in the present study. An additional observation has been that this relationship was most apparent in young patients, those over 35 years of age tending to have frequent conjunctival vessel abnormalities regardless of their ISC count. though sample sizes were small and this finding was not statistically significant. If confirmed in larger groups of patients this observation would be difficult to explain unless repeated but transient vessel obstructions and endothelial damage result in cumulative and permanent vascular damage in the conjunctival vasculature. This hypothesis could be tested by serial observations on the conjunctival vasculature of older patients to confirm whether the pattern of conjunctival vessel anomalies is permanent. and not transient and constantly changing as in younger patients.

In contrast to observations in the conjunctival vasculature no relationship was apparent between the ISC count and abnormal vessels either in the macular area or on the optic disc. Saccular dilatations on the optic disc and perimacular abnormalities were both more common in the high ISC group, but the differences failed to reach statistical significance. There was no evidence that a high ISC count contributed to larger avascular zones at the fovea or in the perimacular area, and although the difference failed to reach significance there was a tendency for the larger avascular zones to occur in the low ISC group. The negative relationship between perimacular vascular abnormalities and the diameter of the foveal avascular zone is explicable if these features represent different stages of the same pathological process. An early vaso-occlusive phase manifested by multiple smallvessel anomalies may progress to total ischaemia and areas previously containing anomalies become replaced by perimacular avascular zones or enlargement of the foveal avascular zone. This is supported by the preponderance of small foveal avascular zones and of high perimacular counts in young patients and also by the elegant serial observations on macular and perimacular vascular remodelling of Asdourian and coworkers. $^{2}$

The failure of the ISC count to correlate with any of the macular abnormalities presumed to result from vaso-occlusion casts doubt on the adequacy of this index in reflecting intravascular sickling in the retinal circulation. This observation conflicts with the association between high ISC counts and vessel anomalies in the conjunctival vasculature, but the latter circulation clearly represents an unusual and specialised capillary bed. It is a predominantly 2dimensional surface circulation exposed to temperature variability and high oxygen tensions atypical of other circulations. It is possible that under these conditions sickled cells which are capable of reverting to the normal discoid shape do so, leaving ISCs as the predominant deformed cell, whereas in other capillary beds vaso-occlusion could be effected by both ISCs and other sickled cells, which, although capable of unsickling in the presence of high oxygen tensions, 
remain deformed at tensions and conditions operative in that capillary bed.

Failure to find a correlation between macular vascular changes and visual acuity reflects partly the mild degree of macular vascular damage in this small sample of patients but also the effects of causes of impaired visual acuity unconnected with macular disease, and possibly also a relative insensitivity in tests of visual acuity. Scores with the Arden gratings varied widely at all ages and in both ISC groups, and fell outside the presumed normal range in over half of this patient group. It is unlikely that these high scores indicate difficulties in communication, understanding, or technical performance of the tests, since satisfactory results have been obtained in population studies in other developing countries. ${ }^{6}$ The frequency of high scores with the Arden gratings may reflect a real decrease in contrast sensitivity in this sample of patients with SS disease, and this is supported by the normal scores obtained in a small comparison group with a normal haemoglobin (AA) genotype. These observations are very preliminary, however, and require further study in large groups of patients and normal people carefully matched for as many determinants as possible.

This study was supported in part by a grant from the Midwest Association for Sickle Cell Anemia (MASCA).

\section{References}

1 Stevens TS. Busse B. Lee C-B. Woolf MB. Galinos SO. Goldberg MF. Sickling hemoglobinopathies: macular and perimacular vascular abnormalities. Arch Ophthalmol 1974; 92: 455-63.

2 Asdourian GK. Nagpal KC. Busse B, et al. Macular and perimacular vascular remodelling in sickling haemoglobinopathies. $\mathrm{Br}$ J Ophthalmol 1976; 60: 431-53.

3 Serjeant GR. Serjeant BE. Condon PI. The conjunctival sign in sickle cell anemia: a relationship with irreversibly sickled cells. JAMA 1972: 219: 1428-31.

4 Arden GB. The importance of measuring contrast sensitivity in cases of visual disturbance. Br J Ophthalmol 1978; 62: 198-209.

5 Arden GB, Jacobson J. A simple grating test for contrast sensitivity: preliminary results indicate value for screening in glaucoma. Invest Ophthalmol Visual Sci 1978; 17: 23-32.

6 Minassian DC. Jones BR. Zargarizadeh A. The Arden grating test of visual function: a preliminary study of its practicability and application in a rural community in north-west Iran. Br J Ophthalmol 1978; 62: 210-2. 